\title{
ARTICLE
}

Received 28 Mar 2015 | Accepted 19 Aug 2015 | Published 25 Sep 2015

DOI: $10.1038 /$ ncomms9396

OPEN

\section{Swarming bacteria migrate by Lévy Walk}

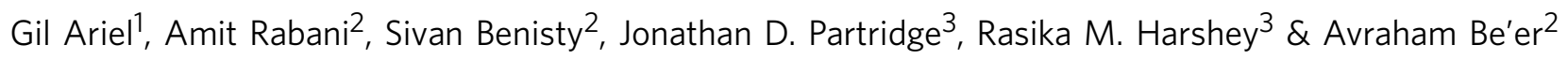

Individual swimming bacteria are known to bias their random trajectories in search of food and to optimize survival. The motion of bacteria within a swarm, wherein they migrate as a collective group over a solid surface, is fundamentally different as typical bacterial swarms show large-scale swirling and streaming motions involving millions to billions of cells. Here by tracking trajectories of fluorescently labelled individuals within such dense swarms, we find that the bacteria are performing super-diffusion, consistent with Lévy walks. Lévy walks are characterized by trajectories that have straight stretches for extended lengths whose variance is infinite. The evidence of super-diffusion consistent with Lévy walks in bacteria suggests that this strategy may have evolved considerably earlier than previously thought.

\footnotetext{
${ }^{1}$ Department of Mathematics, Bar-Ilan University, Ramat Gan 52000, Israel. ${ }^{2}$ Zuckerberg Institute for Water Research, The Jacob Blaustein Institutes for Desert Research, Ben-Gurion University of the Negev, Sede Boqer Campus 84990, Midreshet Ben-Gurion, Israel. ${ }^{3}$ Department of Molecular Biosciences, University of Texas at Austin, Austin, Texas 78712, USA. Correspondence and requests for materials should be addressed to G.A. (email: arielg@math.biu.ac.il).
} 
B acterial swarming is a collective mode of motion in which flagellated bacteria migrate rapidly over surfaces ${ }^{1-6}$. Swarming is typically characterized by densely packed groups of bacteria moving in coherent swirling patterns of whirls and flows that can persist for several seconds ${ }^{7-14}$. There has been considerable recent progress in understanding the swarming phenomenon, including the underlying biological manifestations (cell elongation, increased flagellar density, secretion of wetting agents and increased antibiotic resistance $)^{15-21}$, the physical interactions between cells and the medium (steric and hydrodynamic interactions, and reduction of viscosity in crowded suspensions) ${ }^{7-10,22-24}$, and the statistical properties of the swarm (distribution of group velocities, correlations and clustering) ${ }^{7-10,25-27}$. The swarm traps a water reservoir, within which individual cell speeds are comparable to swimming speeds in bulk liquid ${ }^{2,13,28,29}$. Unlike swimming bacteria, which migrate towards a nutrient source using a biased random walk controlled by a chemosensory signal transduction ${ }^{30}$, the continuously circling motion of individual bacteria within an expanding swarm is apparently random, undirected and independent of the chemotactic signalling system ${ }^{2}$.

Theoretically, it has been shown that dense suspensions of selfpropelled rods are subject to orientational order instabilities which may be driving the vortex-like and irregular dynamic patterns of swarming bacteria ${ }^{31-36}$. In other words, the swirling dynamics is a physical consequence of the mechanical characteristics bacteria exhibit during swarming. None the less, this dynamical pattern seems far from an optimal, energy efficient way to get from one point in the colony to another and the evolutionary advantage of continuously circling in an apparently undirected manner during swarming is not immediately obvious. Current speculations about the advantages of this motion go beyond the simple issue of transit and transportation. For example, it has been suggested that swirling of Bacillus subtilis increases the mixing of oxygen inside suspensions ${ }^{25,37}$. However, these populations are much thicker than typical swarming colonies in which oxygen is not a limiting factor. In addition, swirling was suggested to play a role in prevailing against antimicrobials ${ }^{15,38-40}$.

Previous studies analysing the dynamical swirling patterns of swarming bacteria used one of two experimental approaches. The first used inanimate spherical beads with different diameters $(0.5-10 \mu \mathrm{m})$ that were embedded in the colonies $8,19,41$. The results showed that at length scales of the order of the bead diameter and higher, the motion of the beads is a standard diffusion. This is not surprising, as passive beads within a dense population can be considered as Brownian particles performing a normal diffusion process. The second approach applied video analysis methods (particle image velocimetry or optical flow) ${ }^{8}$ to obtain either short individual trajectories (up to $\sim 1 \mathrm{~s}$; refs $10,13,16$ ) or a locally averaged velocity field describing the collective dynamics of groups and clusters. These methods cannot resolve the individual motion of bacteria to provide long-time trajectories of individuals.

In this work we report a finding that may offer an insight into the swirling patterns of the bacteria. By fluorescently labelling a subset of the motile bacteria, we have tracked individual swarming cells within their natural highly dense context, and obtained long-time trajectories at high spatial and temporal resolution. Our results reveal that the trajectories of swarming cells are super-diffusive, performing a Lévy walk (LW). The LW model is a continuous-time random walk in which particles move with a fixed speed, making sharp turns at random times with a power-law distribution ${ }^{42}$. As a result, these processes are characterized by trajectories that have straight stretches for extended lengths whose variance is infinite $e^{43-45}$. Variations, for example with a distribution of speeds, have also been studied ${ }^{46}$. Extensive research into the properties of LWs has led to the (still debated) hypothesis of its advantage as a foraging and exploration strategy ${ }^{46-58}$. Here we show for the first time observations of swarming bacteria performing random motion consistent with LW behaviour.

\section{Results}

Observation and acquisition of individual cell trajectories. Fluorescently labelled B. subtilis cells expressing a red fluorescence protein (RFP) were mixed with unlabelled cells at a ratio of $\sim$ 1:100 and co-inoculated on swarm agar plates. The bacteria grow into a dense, motile colony, which begins expanding outward after $4 \mathrm{~h}$ and covers the agar plate after a further $3 \mathrm{~h}$. We focused on the outer regions of the expanding swarm where the colony is three-dimensional with multiple layers $(\sim 3 \mu \mathrm{m})$, and the cells are more active (Fig. 1a,b). Single cells migrating within the swarm were detected by fluorescence microscopy (Fig. 1c; Supplementary Movie 1) and their trajectories were acquired at two magnifications (Fig. 1d,e). At high $(\times 63)$ magnification, a single bacterium covers $\sim 1,000$ pixels; this ensures a precise detection of its location and an accurate trajectory. Because the cells tend to leave the field of view within a few seconds, we repeated the experiment using a lower magnification. At low $(\times 20)$ magnification a cell covers $\sim 100$ pixels; this magnification is less precise in resolving shorter spatial scales, however, it allows the capture of longer trajectories. Combining results from both magnifications provided accurate information on the position of the cells as a function of time as well as robust statistics. Similar experiments with a different swarming species, Serratia marcescens, yielded essentially the same results (blue trajectories in Fig. 1d,e).
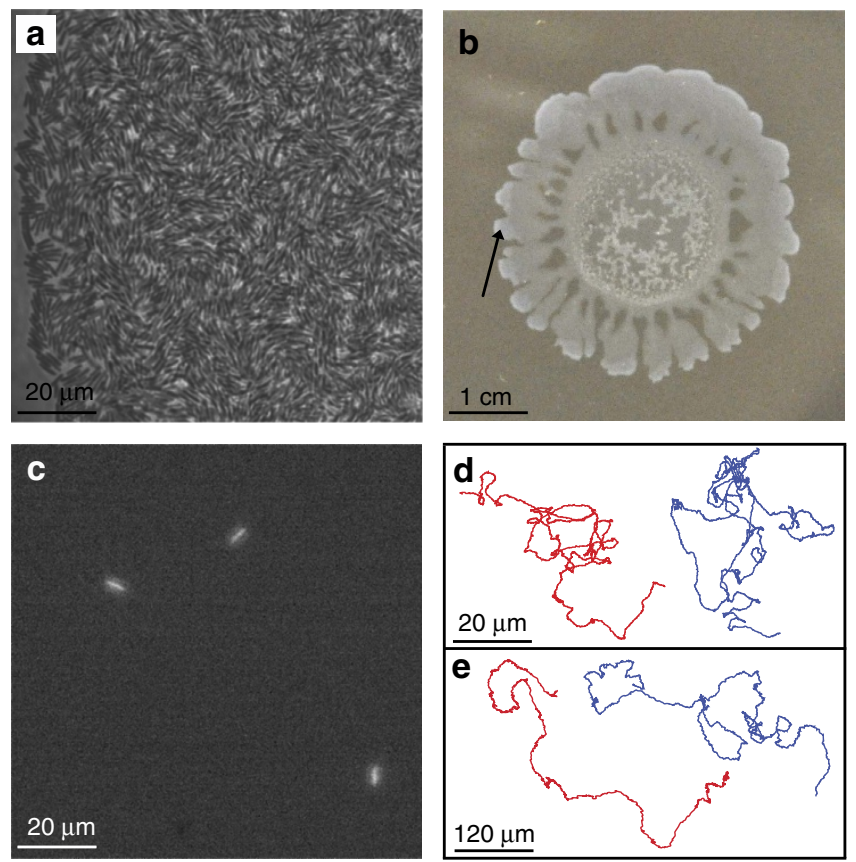

Figure 1 | Tracking individual bacteria within a dense swarm. (a-b) Phase contrast imaging of a wild type B. subtilis swarming colony: at high (a) and low (b) magnifications (region of interest is marked with an arrow in (b)). (c) Fluorescent microscopy showing the fluorescently labelled bacteria only, at high magnification. (d-e) Example trajectories of individual bacteria inside the swarm at high (d) and low (e) magnifications. Left/Red: B. subtilis and Blue/Right: S. marcescens. 
Trajectories are super diffusive. Trajectories of individual swarming bacteria were obtained for a wide range of temporal and spatial scales: from 0.02 to $45 \mathrm{~s}$ and from 0.3 to $400 \mu \mathrm{m}$. Figure 2 shows the mean-square displacement of cells as a function of time. Above a length scale of $\sim 1 \mu \mathrm{m}$ (size of cell; see Fig. 2a), all measurements show supper-diffusive dynamics with an exponent of $\alpha=1.6$,

$$
\left\langle|\mathrm{r}(s+t)-\mathrm{r}(s)|^{2}\right\rangle \sim t^{\alpha},
$$

where $\mathrm{r}(s)$ denotes the location of a cell at time $s$ projected on the focal plane and brackets denote averaging with respect to sample trajectories and time $s$. At longer times $(>2 s)$, the slope reduces to $\sim 1$. This is because cells, which were sampled during a relatively long straight stretch, have a higher probability of leaving the field of view quickly. As a result, long excursions are under-sampled. Figure $2 \mathrm{~b}$ shows that this effect is removed at a lower magnification that allows detection of longer trajectories $(\sim 45 \mathrm{~s}$ and $400 \mu \mathrm{m})$. Combining the two magnifications, we see that the super-diffusive behaviour persists for four orders of spatial and temporal scales. These are considerably larger time and length scales than observed in the collective jets and vortices, which persist typically for $\sim 0.2 \mathrm{~s}$ and have a scale of $\sim 10 \mu \mathrm{m}$. Beyond $400 \mu \mathrm{m}$, the mean-square displacements obtained by different trajectories are significantly different due to a similar bias in the sampling of long trajectories; Supplementary Fig. 1. In addition, the variance in the apparent diffusion constants (the intersection point of curves with the $y$ axis) seems to be due to sampling bias rather than a property of individual bacteria; Supplementary Fig. 2.

Trajectories are consistent with Lévy walks. To further understand the bacterial dynamics inside the swarm and compare it with previous results of the global swarm or bead dynamics, we analysed additional aspects of the trajectories. Since the type of dynamics that cells follow is mostly governed by their long-time asymptotic properties, low magnification data, which captures longer trajectories, was used. Figure $3 \mathrm{a}$ shows the density of displacements (displacement of cells between a fixed number of frames), $P(\Delta x, \Delta t)$, using the lower magnification. Assuming a scaling of displacements as $\Delta t^{1 / \beta}$, we find that $\beta=1.27$ minimizes the cumulative variance between the four times depicted in Fig. 3a $(\Delta t=1,5,10$ and 40$)$, in agreement with the theoretical prediction of $\alpha+\beta=3$ for LWs. In addition, the scaled displacement density fits well a symmetric Lévy stable distribution with stability parameter $\beta$, scale parameter 5.2 and zero location parameter
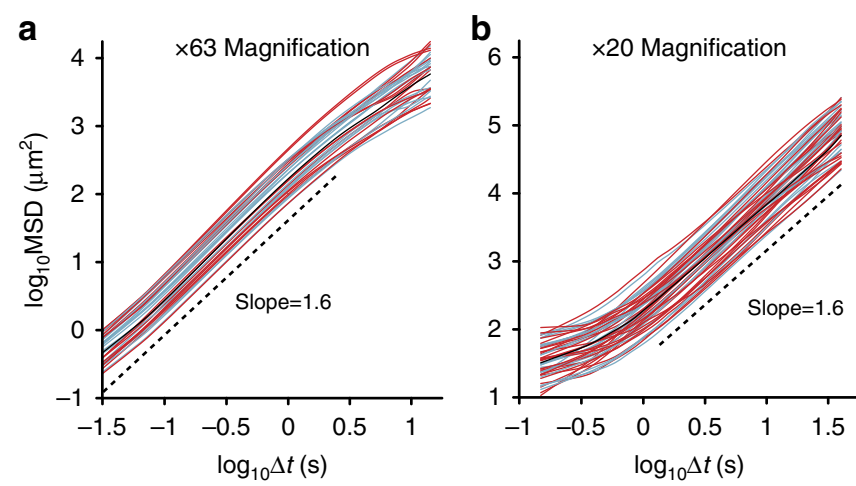

Figure 2 | Mean square displacement of single bacteria. A slope of 1.6 is obtained for all bacteria; red lines show results with $B$. subtilis and blue lines with S. marcescens. The black line is the average of all bacteria. Data obtained with (a) high and (b) low magnifications. (zero shift; see black line). By comparison, fitting the scaled displacement distribution to a Gaussian yields a poor approximation (grey line). However, due to insufficient sampling, it is difficult to ascertain the power-law decay of the tail (Supplementary Fig. 3). The symmetric, centralized distribution implies that there is no mean drift, which is consistent with Fig. 3b, showing a uniform distribution of directions. This verifies that there is no globally preferred direction (Supplementary Fig. 4). Figure 3c shows that the direction of motion remains fairly constant for times that are significantly longer than the characteristic run times in bacteria $(\sim 1 \mathrm{~s})$. In addition, Fig. 3d depicts the velocity auto-correlation function, $C(\Delta t)=\langle\mathrm{v}(t) \cdot \mathrm{v}(t+\Delta t)\rangle$ obtained with the lower magnification data. Velocities decay as $\Delta t^{-\delta}$ with $\delta=0.41$, in agreement with the theory of LWs, predicting that $\delta=2-\alpha$ (Supplementary Note 1). The fit to an exponential is poor (Supplementary Fig. 5).

Next, we tested the hypothesis of a LW model by defining 'turning points' in the trajectories of cells as an instant with angular speed $\omega$ larger than some threshold (following some smoothing of trajectories to eliminate jitter in movies; see Methods for details). As turning is a short-time and local event, high magnification data, which is captured at high temporal and spatial resolution, was used. Figure 4a shows a typical trajectory of a cell with turning points (marked in red) defined using $\omega=10 \mathrm{rad} \cdot \mathrm{s}^{-1}\left(\sim 60^{\circ}\right.$ in $\left.0.1 \mathrm{~s}\right)$. The distance between two consequent turns can be considered as a 'walking segment' in a LW. Figure $4 \mathrm{~b}$ shows the length $\Delta L$ of walking segments as a function of its duration, $\Delta t$, indicating an approximately constant
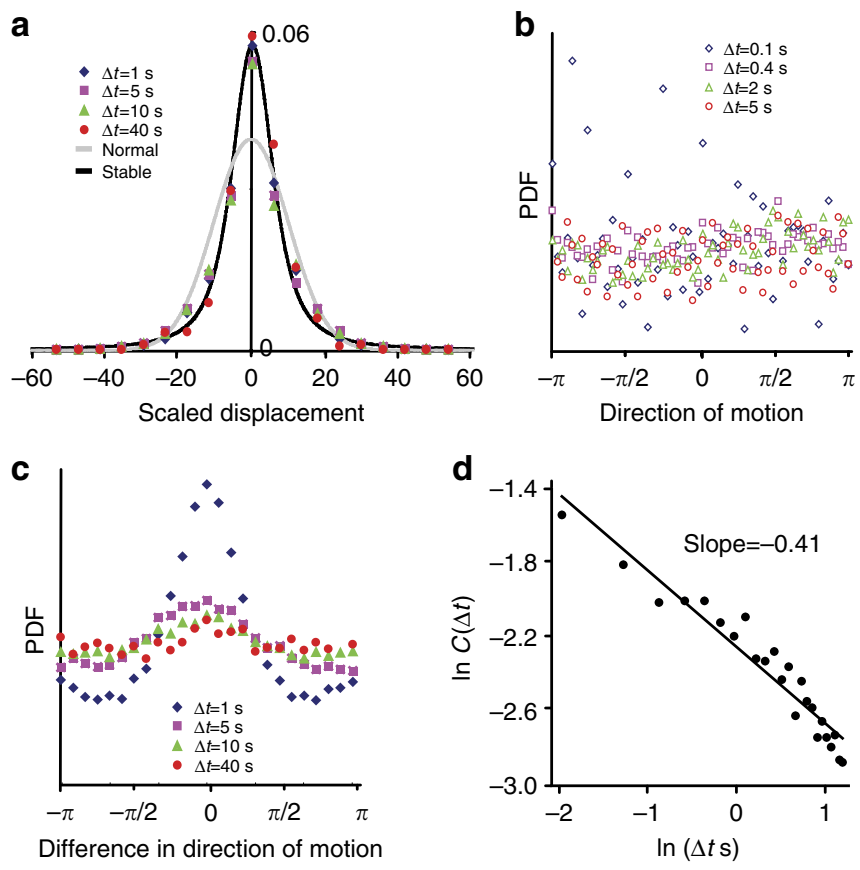

Figure 3 | Statistics of cell displacements. Analysis of trajectories using the low magnification. (a) The distribution of cell displacements along each axis between a fixed number of frames. Following a scaling of $\Delta t^{1 / 1.33}$, all distributions approximately fit a Lévy stable distribution with parameter 1.33. (b) The probability density function (PDF) of cell directions (with respect to an arbitrary lateral axis of the frame) is uniform for all time intervals. Data were taken at high magnification. (c) Persistence in the direction of motion. The direction of motion remains fairly constant for times that are significantly longer than the characteristic run times in bacteria ( $\sim \mathrm{s}$ ). Experiments with S. marcescens yield similar results (data not shown). (d) The velocity auto-correlation function decays algebraically with slope of -0.41 , in agreement with the theory of LWs. 

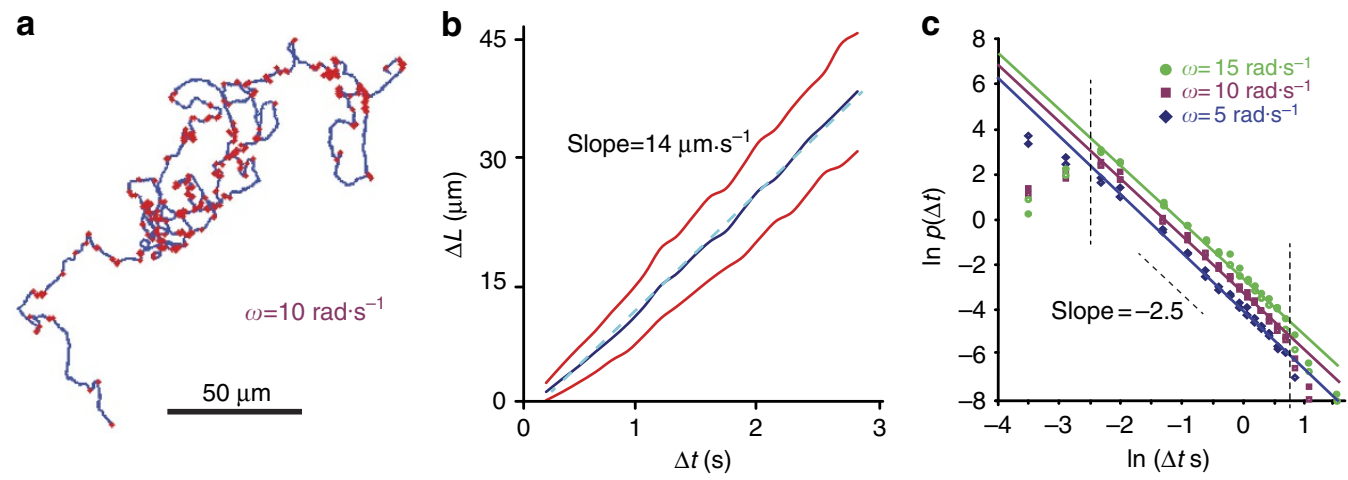

Figure 4 | Bacterial trajectories have Lévy walk characteristics. Analysis using the high magnification. (a) A typical trajectory of a B. subtilis bacterium with turning points marked in red. Trajectory was obtained at the high magnification. (b) The length $\Delta L$ of walking segments as a function of its duration $\Delta t$, indicating an approximately constant speed. A similar plot was obtained for $S$. marcescens cells with the same average speed. (c) Distribution of waiting times between turns showing a power-law decay with a slope of -2.5 , in agreement with the theory of LWs. Filled circles, squares and diamonds show results with B. subtilis; empty circles, squares and diamonds show S. marcescens. The slope is independent of the cutoff $\omega$. Straight lines are least squares fits. At short and long times (indicated by a vertical dashed line), the fit to a power-law deteriorates.

speed. The distribution of speeds within segments is plotted in Supplementary Fig. 6 .

These data (Fig. 4b) imply that bacteria perform a LW rather than a Lévy flight. In contrast to the LW model, a Lévy flight is a jump process in which particle speeds vary significantly, occasionally making fast and long displacements. Trajectories of both models are indistinguishable. Indeed, Fig. $4 \mathrm{c}$ shows the tail of the density of segment lengths that decays like $\Delta t^{-\gamma}$ with $\gamma=2.5$ regardless of the choice of cutoff $\omega$. See Supplementary Fig. 7 for results obtained using the low magnification data. Using the Akaike Information Criterion to quantitatively compare the relative likelihood of a power-law model to an exponential tail ${ }^{56}$ yields a weight of practically one in favour of the power-law model. This is in excellent agreement with the theory of LWs as a continuous-time random walk with constant speed, predicting that $\alpha+\gamma=4$ (refs 59,60).

Overall, our findings exclude stochastic models showing super-diffusion other than LW, for example, fractional Brownian motion ${ }^{46,61}$, generalized Langevin equations $s^{46,62,63}$, correlated and persistent random walks ${ }^{46,53-55,57,58,64}$, persistent random walks with variable persistence times ${ }^{65}$ and Lévy flights ${ }^{42,61}$. See Supplementary Notes $2-8$ for supported details and simulation results in Supplementary Figs 8-13.

\section{Discussion}

In nature, organisms face harsh conditions in which nutrients and other essential necessary resources may be depleted. In the absence of information, moving individuals resort to various random search strategies, depending on their movement abilities, the environment and the type of resources sought.

Swimming bacteria move by a process called run-and-tumble, in which short random movements (tumbles) are interspersed by long trajectories (runs). A chemotaxis signalling network encodes a short-term memory that allows the bacteria to control the length of runs and therefore bias their motion towards nutrients or away from repellents ${ }^{30}$. Bartumeus and Levin ${ }^{66}$ hypothesized that individual swimming bacteria may be performing a LW due to a power-law (with a cutoff) distribution of run times ${ }^{67,68,69}$. However, several recent experimental works revealed that these bacteria essentially follow a standard random walk (normal diffusion $)^{70-72}$. Thus, single cells cannot improve their search strategy beyond this limitation.

We have shown that the bacteria examined in this study can use the collective dynamics of the swarm to fundamentally change the statistical properties of their dynamics. In particular, our results suggest that bacteria perform a LW. LWs were found to optimize searching in sparsely and randomly distributed targets in the absence of memory ${ }^{47,48}$. Although we cannot conclude that the random walk in our system is used as a search strategy, it is possible that swarming bacteria use the LW towards a similar end, which would imply a different foraging mechanism than that controlled by the chemosensory system during swimming. Our study shows that swarming bacteria are somehow using their large numbers to fundamentally change the statistical properties of their collective motion into a LW dynamics. This finding is in keeping with our earlier observation that $\mathrm{MgO}$ particles deposited on the surface of the swarm fluid display super-diffuse trajectories ${ }^{19}$. The high energy cost required to maintain the swirling in the swarm must be justified if it helps override the threat of death from starvation or from environmental hazards. In addition, such a behaviour has biological applications in food foraging as well as genetic and phenotypic spreading in the cases of wound repair and cancer invasion in vitro ${ }^{73,74}$, and for understanding fundamental aspects of how neighbouring individuals from the same population eventually end up in completely different locations.

Any physical or biological realization of a mathematical model is never precise. Accordingly, the observed super-diffusion deviates in some aspects from those of the mathematical LW. This suggests that other physical mechanisms may be important, in particular at very short or long-time scales. However, our finding suggest that in a wide range of 3-4 temporal and spatial orders of magnitude, the dynamics of swarming bacteria is consistent with a LW.

The mechanisms underlying the super-diffusive behaviour we report during swarming are fundamentally different than those hypothesized for swimming bacteria ${ }^{66-69}$. Numerous experimental and theoretical works analysing and describing the flow patterns and physical mechanisms underlying swarming show that the velocity of a swarming cell is mostly governed by the collective dynamics of the swarm and the fluid it carries rather than the precise operation of the individual flagella ${ }^{8-36}$. Thus, a bacterium does not 'decide' to move as a Levy walker by controlling the frequency of tumbles. Instead, we suggest that it is the collective flow of the entire swarm that facilitates the LW. In this respect, the mechanism for LW is different than in other complex organisms which have been reported to follow a $\mathrm{LW}^{46,58}$. Indeed, we observe that our results are in accordance with super-diffusion reported for laminar fluid flow in a rotating 
annulus ${ }^{75}$. Solomon et al. ${ }^{75}$ suggested that particles 'stick' to temporarily invariant surfaces around vortices for durations that exhibit power-law decays with an exponent of $\gamma=2.3$. The variance in the azimuthal displacement also shows a power-law growth with $\alpha=1.65$. The agreement with our experimental results $(\alpha=1.6, \gamma=2.5)$ suggests a possible mechanism for superdiffusion in swarming bacteria - that the swirling and vortex-like patterns created by the orientational instabilities of swarming bacteria plays a similar role as the rotating two-dimensional flow and that bacteria 'get trapped' in local vortices between extended extrusions along jets ${ }^{59}$. The difference between inanimate particles and swarming bacteria is that in a bacterial swarm, the turbulent velocity field is due to instabilities caused, in part, by the constant injection of energy using flagella ${ }^{31-36}$. We hypothesize that bacteria have optimized this strategy for surface translocation, perhaps by suppressing chemotaxis, and possibly to exploit it as a search strategy.

\section{Methods}

Bacterial strains and growth protocol. We have performed the experiments using two robust swarming bacteria, yielding essentially the same results. The first was $B$. subtilis strain 3610 (wild type), which is a Gram positive rod-shaped $(0.8 \times 5 \mu \mathrm{m})$ species, used as a model system in many quantitative swarming experiments ${ }^{1-4,7-10,15,19}$. The cells were grown on agar plates $\left(\mathrm{gl}^{-1}\right.$ peptone and $0.5 \%$ agar at $30{ }^{\circ} \mathrm{C}$ ); cells formed dense colonies (thickness of 3-4 $\mu \mathrm{m}$ ) and began expanding outwards around $4 \mathrm{~h}$ after inoculation. Note that these conditions are different from published protocols that use Luria Broth (LB) and where cells swarm in a monolayer and expand out earlier ${ }^{1}$. A second derivative strain of 3610 was labelled with red fluorescent protein or RFP, where the protein was expressed from a chromosomal location ( $p p s B::$ PtrpE-mCherry). The wild type were mixed with the RFP variant (at a ratio of 100:1) in a small tube before inoculation, then co-inoculated on swarm agar plates. Labelling does not affect swarming behaviour (Supplementary Tables 1-2). Under fluorescent microscopy only RFP cells are seen, which enables the precise detection of single cells trajectories even in a highly crowded population. The second bacterial system was $S$. marcescens strain 274 (wild type), which is a Gram negative rod-shaped $(0.8 \times 4 \mu \mathrm{m})$ species, used as a model system in previous swarming experiments $s^{1,15,19,27}$. The cells were grown on agar plates (LB and $0.5 \%$ agar at $30^{\circ} \mathrm{C}$ ); cells formed dense colonies (thickness of $3-4 \mu \mathrm{m}$ ), began expanding outwards around $5 \mathrm{~h}$ after inoculation and swarmed rapidly. For tracking individuals, the $S$. marcescens were labelled with a green fluorescent protein (GFP) expressed from a plasmid (pTRC99a::GFP; strain JP1020). As described above for B. subtilis, wild type and GFP-labelled strains were mixed at a ratio of 100:1 before inoculation on agar plates. All bacteria were stored at $-80^{\circ} \mathrm{C}$ in $50 \%$ glycerol stocks (antibiotics was added to frozen stocks of the RFP and GFP mutants; phleomycin for B. subtilis $\left(7 \mu \mathrm{g} \mathrm{ml}^{-1}\right)$ and ampicillin $\left(100 \mu \mathrm{g} \mathrm{ml}^{-1}\right)$ for S. marcescens) and grown overnight in LB broth at $30^{\circ} \mathrm{C}$ and shaking (200 r.p.m.) before plate inoculation ( $5 \mu \mathrm{l}$ at the centre of each plate).

Observations. Optical microscopy (Zeiss Axio Imager Z2; $\times 20$, and $\times 63$ lenses), equipped with a sensitive high resolution video camera (NEO, Andor), was used to capture the motion of the labelled cells under fluorescence microscopy (100 frames per sec at $\times 63$ and 7 frames per sec at $\times 20$ and $1,000 \times 1,000$ pixels for both magnifications). Trajectories were obtained and analysed using Matlab. For both bacterial species, no photobleaching was observed during acquisition times (two minutes for each experiment; 6,000 frames). In each field of view we typically had $\sim 5$ labelled cells at $\times 63$ and $\sim 50$ labelled cells at $\times 20$. The total data summarizes results from tens of experiments with hundreds of cells from each species. Because standard fluorescent light strongly affects cell motility (it usually completely stops their motion in less than $1 \mathrm{~s}$ ), we used a slightly modified version for the filters and dichroic mirror. The GFP-labelled cells were observed by standard yellow fluorescent protein Zeiss illumination setup instead of the standard GFP one (Filter set 46 yellow fluorescent protein shift free: Excitation 500/25; Beam Splitter 515; Emission 535/30). The cell intensity was slightly weaker compared to GFP. The RFP labelled cells, designed initially for mCherry illumination setup, were observed by standard Rhodamin (RFP) Zeiss illumination setup (Filter set 20 Rhodamin shift free: Excitation 546/12; Beam Splitter 560; Emission 607/80).

Smoothing of trajectories. In order to define turning points, trajectories were smoothed using Matlab's malowess function which locally fits a polynomial (second order) to a moving window (11 frames). Then, 'turning points' were defined as an instant with angular speed larger than a given threshold.

\section{References}

1. Harshey, R. M. Bacterial motility on a surface: many ways to a common goal. Annu. Rev. Microbiol. 57, 249-273 (2003).
2. Partridge, J. D. \& Harshey, R. M. Swarming: flexible roaming plans. J. Bacteriol. 195, 909-918 (2013)

3. Kearns, D. B. \& Losick, R. Swarming motility in undomesticated Bacillus subtilis. Mol. Microbiol. 49, 581-590 (2004).

4. Kearns, D. B. A field guide to bacterial swarming motility. Nat. Rev. Microbiol. 8, 634-644 (2010).

5. Copeland, M. F. \& Weibel, D. B. Bacterial Swarming: A model system for studying dynamic self-assembly. Soft Matter 5, 1174-1187 (2009).

6. Ariel, G., Shklarsh, A., Kalisman, O., Ingham, C. \& Ben-Jacob, E. From organized internal traffic to collective navigation of bacterial swarms. New J. Phys. 15, 125019 (2013).

7. Benisty, S., Ben-Jacob, E., Ariel, G. \& Be'er, A. Antibiotic-induced anomalous statistics of collective bacterial swarming. Phys. Rev. Lett. 114, 018105 (2015).

8. Zhang, H. P., Be'er, A., Smith, R. S., Florin, E.-L. \& Swinney, H. L. Swarming dynamics in bacterial colonies. Europhys. Lett. 87, 48011 (2009).

9. Zhang, H. P., Be'er, A., Florin, E.-L. \& Swinney, H. L. Collective motion and densityfluctuations in bacterial colonies. Proc. Natl Acad. Sci. USA 107, 13626-13630 (2010).

10. Chen, X., Dong, X., Be'er, A., Swinney, H. L. \& Zhang, H. P. Scale-invariant correlations in dynamic bacterial clusters. Phys. Rev. Lett. 108, 148101 (2012).

11. Be'er, A. et al. Paenibacillus dendritiformis bacterial colony growth depends on surfactant but not on bacterial motion. J. Bacteriol. 191, 5758-5764 (2009).

12. Be'er, A., Strain, S. K., Hernandez, R. A., Ben-Jacob, E. \& Florin, E.-L. Periodic reversals in Paenibacillus dendritiformis swarming. J. Bacteriol. 195, 2709-2717 (2013).

13. Darnton, N. C., Turner, L., Rojevsky, S. \& Berg, H. C. Dynamics of bacterial swarming. Biophys. J. 98, 2082-2090 (2010).

14. Ingham, C. J. \& Ben-Jacob, E. Swarming and complex pattern formation in Paenibacillus vortex studied by imaging and tracking cells. BMC Microbiol. 8, 36 (2008).

15. Butler, M. T., Wang, Q. \& Harshey, R. M. Cell density and mobility protect swarming bacteria against antibiotics. Proc. Natl Acad. Sci. USA 107, 3776-3781 (2010).

16. Copeland, M. F., Flickinger, S. T., Tuson, H. H. \& Weibel, D. B. Studying the dynamics of flagella in multicellular communities of Escherichia coli by using biarsenical dyes. Appl. Environ. Microbiol. 76, 1241-1250 (2010).

17. Tuson, H. H., Copeland, M. F., Carey, S., Sacotte, R. \& Weibel, D. B. Flagellum density regulates Proteus mirabilis swarmer cell motility in viscous environments. J. Bacteriol. 195, 368-377 (2013).

18. Patrick, J. E. \& Kearns, D. B. MinJ (YvjD) is a topological determinant of cell division in Bacillus subtilis. Mol. Microbiol. 70, 1166-1179 (2008).

19. Be'er, A. \& Harshey, R. M. Collective motion of surfactant-producing bacteria imparts superdiffusivity to their upper surface. Biophys. J. 101, 1017-1024 (2011).

20. Turner, L., Zhang, R., Darnton, N. C. \& Berg, H. C. Visualization of flagella during bacterial swarming. J. Bacteriol. 192, 3259-3267 (2010).

21. Roth, D. et al. Identification and characterization of a highly motile and antibiotic refractory subpopulation involved in the expansion of swarming colonies of Paenibacillus vortex. Environ. Microbiol. 15, 2532-2544 (2013).

22. Sokolov, A., Aranson, I., Kessler, J. \& Goldstein, R. Model for dynamical coherence in thin films of self-propelled microorganisms. Phys. Rev. Lett. 98, 158102(R) (2007).

23. Cisneros, L. H., Cortez, R., Dombrowski, C., Goldstein, R. E. \& Kessler, J. O. Fluid dynamics of self-propelled microorganisms, from individuals to concentrated populations. Exp. Fluids 43, 737-753 (2007).

24. Sokolov, A. \& Aranson, I. S. Reduction of viscosity in suspension of swimming bacteria. Phys. Rev. Lett. 103, 148101 (2009).

25. Sokolov, A. \& Aranson, I. S. Physical properties of collective motion in suspensions of bacteria. Phys. Rev. Lett. 109, 248109 (2012).

26. Dunkel, J. et al. Fluid dynamics of bacterial turbulence. Phys. Rev. Lett. 110, 228102 (2013)

27. Rabani, A., Ariel, G. \& Be'er, A. Collective motion of spherical bacteria. PLoS ONE 8, e83760 (2013)

28. Wang, Q., Suzuki, A., Mariconda, S., Porwollik, S. \& Harshey, R. M. Sensing wetness: a new role for the bacterial flagellum. $E M B O ~ J . ~ 24,2034-2042$ (2005).

29. Wu, Y., Hosu, B. G. \& Berg, H. C. Microbubbles reveal chiral fluid flows in bacterial swarms. Proc. Natl Acad. Sci. USA 108, 4147-4151 (2011).

30. Berg, H. C. E. coli in motion (Springer Science \& Business Media, U.S.A., 2004)

31. Wensink, H. H. et al. Meso-scale turbulence in living fluids. Proc. Natl Acad. Sci. USA 109, 14308-14313 (2012).

32. Saintillan, D. \& Shelley, M. J. Orientational order and instabilities in suspensions of self-locomoting rods. Phys. Rev. Lett. 99, 058102 (2007).

33. Simha, R. A. \& Ramaswamy, S. Hydrodynamic fluctuations and instabilities in ordered suspensions of self-propelled particles. Phys. Rev. Lett. 89, 058101 (2002).

34. Wolgemuth, C. W. Collective swimming and the dynamics of bacterial turbulence. Biophys. J. 95, 1564-1574 (2008). 
35. Sambelashvili, N., Lau, A. W. C. \& Cai, D. Dynamics of bacterial flow: Emergence of spatiotempral coherent structures. Phys. Lett. A. 360, 507-511 (2007).

36. Sankararaman, S. \& Ramaswamy, S. Instabilities and waves in thin films of living fluids. Phys. Rev. Lett. 102, 118107 (2009).

37. Sokolov, A., Goldstein, R. E., Feldchtein, F. I. \& Aranson, I. S. Enhanced mixing and spatial instability in concentrated bacterial suspensions. Phys. Rev. E 80, 031903 (2009).

38. Lai, S., Tremblay, J. \& Déziel, E. Swarming motility: a multicellular behaviour conferring antimicrobial resistance. Environ. Microbiol. 11, 126-136 (2009).

39. Kim, W., Killam, T., Sood, V. \& Surette, M. G. Swarm-cell differentiation in Salmonella enterica serovar typhimurium results in elevated resistance to multiple antibiotics. J Bacteriol. 185, 3111-3117 (2003).

40. Overhage, J., Bains, M., Brazas, M. D. \& Hancock, R. E. Swarming of Pseudomonas aeruginosa is a complex adaptation leading to increased production of virulence factors and antibiotic resistance. J Bacteriol. 190, 2671-2679 (2008)

41. Xiao-Lun, W. \& Libchaber, A. Particle diffusion in a quasi-two-dimensional bacterial bath. Phys. Rev. Lett. 84, 3017-3020 (2000).

42. Shlesinger, M. F., Klafter, J. \& Wong, Y. M. Random walks with infinite spatial and temporal moments. J. Stat. Phys. 77, 499-512 (1982).

43. Klafter, J., Shlesinger, M. F. \& Zumofen, G. Beyond Brownian motion. Phys. Today 49, 33-39 (1996).

44. Bouchaud, J. P. \& Georges, A. Anomalous diffusion in disordered media: statistical mechanisms, models and physical applications. Phys. Rep. 195, 127-293 (1990).

45. Klafter, J. \& Zumofen, G. Lévy statistics in a Hamiltonian system. Phys. Rev. E 49, 4873-4877 (1994).

46. Metzler, R., Jeon, J. H., Cherstvy, A. G. \& Barkai, E. Anomalous diffusion models and their properties: non-stationarity, non-ergodicity, and ageing at the centenary of single particle tracking. Phys. Chem. Chem. Phys. 16, 24128-24164 (2014).

47. Bartumeus, F., da Luz, M. G. E., Viswanathan, G. M. \& Catalan, J. Animal search strategies: a quantitative random-walk analysis. Ecology 86, 3078-3087 (2005).

48. Bartumeus, F. Lévy processes in animal movement: an evolutionary hypothesis. Fractals. 15, 151-162 (2007).

49. Humphries, N. E. \& Sims, D. W. Optimal foraging strategies: Lévy walks balance searching and patch exploitation under a very broad range of conditions. J. Theor. Biol. 358, 179-193 (2014).

50. Viswanathan, G. M. et al. Optimizing the success of random searches. Nature 401, 911-914 (1999).

51. Viswanathan, G. M., Raposo, E. P. \& da Luz, M. G. E. Lévy flights and superdiffusion in the context of biological encounters and random searches. Phys. Life Rev. 5, 133-150 (2008).

52. Raichlen, D. A. et al. Evidence of Levy walk foraging patterns in human huntergatherers. Proc. Natl Acad. Sci. USA 111, 728-733 (2014).

53. Reynolds, A. M. \& Rhodes, C. J. The Lévy flight paradigm: random search patterns and mechanisms. Ecology 90, 877-887 (2009).

54. Reynolds, A. M. Bridging the gulf between correlated random walks and Lévy walks: autocorrelation as a source of Lévy walk movement patterns. J. R. Soc. Interface 7, 1753-1758 (2010).

55. Harris, T. H. et al. Generalized Lévy walks and the role of chemokines in migration of effector CD8 + T cells. Nature 486, 545-549 (2012).

56. Edwards, A. M. et al. Revisiting Lévy flight search patterns of wandering albatrosses, bumblebees and deer. Nature 449, 1044-1048 (2007).

57. Codling, E. A., Plank, M. J. \& Benhamou, S. Random walk models in biology. J. R. Soc. Interface 5, 813-834 (2008).

58. Benhamou, S. How many animals really do the lévy walk? Ecology 88, 1962-1969 (2007).

59. Clauset, A., Shalizi, C. R. \& Newman, M. E. Power-law distributions in empirical data. SIREV 51, 661-703 (2009)

60. Zaburdaev, V., Denisov, S. \& Klafter, J. Lévy walks. Rev. Mod. Phys. 87, 483 (2015).

61. Mandelbrot, B. \& van Ness, J. W. Fractional Brownian motions, fractional noises and applications. SIAM Review 10, 422-437 (1968).

62. Eule, S., Zaburdaev, V., Friedrich, R. \& Geisel, T. Langevin description of superdiffusive Lévy processes. Phys. Rev. E. 86, 041134 (2012).
63. Fox, R. F. The generalized Langevin equation with Gaussian fluctuations. J. Math. Phys. 18, 2331 (1977).

64. Renshaw, E. \& Henderson, R. The correlated random walk. J. App. Prob. 18, 403-414 (1981).

65. Petrovskii, S., Mashanova, A. \& Jansen, V. A. Variation in individual walking behavior creates the impression of a Lévy flight. Proc. Natl Acad. Sci. USA 108, 8704-8707 (2011).

66. Bartumeus, F. \& Levin, S. A. Fractal reorientation clocks: linking animal behavior to statistical patters of search. Proc. Natl Acad. Sci. USA 105, 19072-19077 (2008).

67. Korobkova, E., Emont, T., Vilar, J. M. G., Shimizu, T. \& Cluzel, P. From molecular noise to behavioural variability in a single bacterium. Nature 428, 574-578 (2004).

68. Sneddon, M. W., Pontius, W. \& Emonet, T. Stochastic coordination of multiple actuators reduces latency and improves chemotactic response in bacteria. Proc Natl Acad. Sci. USA 109, 805-810 (2012).

69. Tu, Y. \& Grinstein, G. How white noise generates power-law switching in bacterial flagellar motors. Phys. Rev. Lett. 94, 208101 (2005).

70. Wu, M., Roberts, J. W., Kim, S., Koch, D. L. \& DeLisa, M. P. Collective bacterial dynamics revealed using a three-dimensional population-scale defocused particle tracking technique. Appl. Environ. Microbiol. 72, 4987-4994 (2006).

71. Saragosti, J., Silberzan, P. \& Buguin, A. Modeling E. coli tumbles by rotational diffusion. Implications for chemotaxis. PLoS ONE 7, e35412 (2012).

72. Turner, L., Ryu, W. S. \& Berg, H. C. Real-time imaging of fluorescent flagellar filaments. J. Bacteriol. 182, 2793-2801 (2000).

73. Zaritsky, A. et al. Propagating waves of directionality and coordination orchestrate collective cell migration. PLoS ONE 10, e1003747 (2014).

74. Zaritsky, A., Natan, S., Ben-Jacob, E. \& Tsarfaty, I. Emergence of HGF/SF-induced coordinated cellular motility. PLoS ONE 7, e44671 (2012)

75. Solomon, T. H., Weeks, E. R. \& Swinney, H. L. Observation of anomalous diffusion and Lévy flights in a two-dimensional rotating flow. Phys. Rev. Lett. 71, 3975-3978 (1993).

\section{Acknowledgements}

We thank E. Barkai for valuable discussions, ideas, suggestions and informal meetings. We thank D.B. Kearns for the generous gift of B. subtilis and for many useful discussions We thank A. Eldar and S. Pollak for creating the RFP mutants. We thank D. Roth for ideas and comments. R.M.H is thankful for partial support from the National Institutes of Health Grant GM112507, and the Robert Welch Foundation Grant F-1811. A.B. is thankful for partial support from an EU/FP7 REA grant 321777, The Israel Science Foundation (Grant No. 337/12), and the Roy J. Zuckerberg Career Development Chair for Water Research. We are grateful to two anonymous referees for numerous ideas, comments and corrections that considerably improved the paper. The paper is dedicated to the memory of our mentor, colleague and friend Eshel Ben-Jacob.

\section{Author contributions}

G.A. and A.B. directed the research, designed the experiments, performed the experiments and wrote the manuscript. R.M.H. designed the experiments and wrote the manuscript. J.D.P., A.R. and S.B. performed the experiments.

\section{Additional information}

Supplementary Information accompanies this paper at http://www.nature.com/ naturecommunications

Competing financial interests: The authors declare no competing financial interests.

Reprints and permission information is available online at http://npg.nature.com/ reprintsandpermissions/

How to cite this article: Ariel, G. et al. Swarming bacteria migrate by Lévy Walk. Nat Commun. 6:8396 doi: 10.1038/ncomms9396 (2015).

This work is licensed under a Creative Commons Attribution 4.0 International License. The images or other third party material in this article are included in the article's Creative Commons license, unless indicated otherwise in the credit line; if the material is not included under the Creative Commons license, users will need to obtain permission from the license holder to reproduce the material. To view a copy of this license, visit http://creativecommons.org/licenses/by/4.0/ 